\title{
Utility of continuous glucose monitoring following gastrectomy
}

\author{
Takeshi Kubota $^{1}$ [ $\cdot$ Katsutoshi Shoda ${ }^{1} \cdot$ Emi Ushigome $^{2} \cdot$ Toshiyuki Kosuga $^{1} \cdot$ Hirotaka Konishi $^{1} \cdot$ Atsushi Shiozaki $^{1}$. \\ Michihiro Kudo $^{1}$ - Tomohiro Arita ${ }^{1}$ - Yasutoshi Murayama ${ }^{1} \cdot$ Ryo Morimura $^{1} \cdot$ Hisashi Ikoma ${ }^{1}$. Yoshiaki Kuriu ${ }^{1}$. \\ Masayoshi Nakanishi ${ }^{1} \cdot$ Hitoshi Fujiwara ${ }^{1} \cdot K_{\text {Kazuma Okamoto }}{ }^{1} \cdot$ Michiaki Fukui $^{2} \cdot$ Eigo Otsuji $^{1}$
}

Received: 9 August 2019 / Accepted: 27 December 2019 / Published online: 8 January 2020

(c) The International Gastric Cancer Association and The Japanese Gastric Cancer Association 2020

\begin{abstract}
Background Glucose fluctuation after gastrectomy represented by dumping syndrome is a well-known post-gastrectomy syndrome that negatively impacts patient quality of life. However, the current methods of post-gastrectomy glucose monitoring do not comprehensively capture the postoperative blood glucose fluctuations that characterize this.

Methods We used a continuous glucose monitoring (CGM) system to document the glycemic profiles of patients undergoing gastrectomy and compared these between patients undergoing distal gastrectomy (DG) and total gastrectomy (TG). To evaluate post-gastrectomy syndromes, including dumping syndrome, we used the Post-gastrectomy Syndrome Assessment Scale 37-item questionnaire. The glycemic profiles were also compared using this tool.

Results We studied 57 patients who had undergone DG and 13 who had undergone TG between September 2017 and September 2019. Our results revealed larger diurnal glycemic variability and longer periods of nocturnal hypoglycemia after gastrectomy. The dumping score was worse in the TG than in the DG group (TG $2.4 \pm 1.4 v s$. DG 1.3 $\pm 1.2, P=0.0061$ ). Importantly, 30 of $57 \mathrm{DG}$ patients (52.6\%) and 5 of $13 \mathrm{TG}$ patients (38.5\%) experienced postprandial hypoglycemia following hyperglycemia without hypoglycemic symptoms. There was no correlation between the dumping symptom score and glycemic variability $(\rho=0.0545, P=0.6662)$.

Conclusions CGM demonstrated diurnal glycemic variability and nocturnal hypoglycemia in patients undergoing gastrectomy. Because some hypoglycemic patients did not develop symptoms and glycemic variability was not necessarily associated with dumping symptom, dumping syndrome must only partially explain the postoperative glucose fluctuations.
\end{abstract}

Keywords Gastric cancer · Gastrectomy · Continuous glucose monitoring · Glycemic variability · Dumping syndrome

\section{Introduction}

Gastric cancer is the fifth most common type of cancer and the third most common cause of cancer-related death worldwide [1]. Surgical resection remains the only curative treatment option and regional lymphadenectomy is recommended as a part of radical gastrectomy. However, gastrectomy disrupts reservoir capacity, mechanical digestion, and

Takeshi Kubota

tkubot@koto.kpu-m.ac.jp

1 Division of Digestive Surgery, Department of Surgery, Kyoto Prefectural University of Medicine, 465 Kajii-cho, Kawaramachi-Hirokoji, Kamigyo-ku, Kyoto 602-8566, Japan

2 Department of Endocrinology and Metabolism, Kyoto Prefectural University of Medicine, 465 Kajii-cho, Kawaramachi-Hirokoji, Kamigyo-ku, Kyoto 602-8566, Japan gastric emptying. Because of the altered form and function of the stomach, various post-gastrectomy syndromes can occur. One of these, dumping syndrome is a common, wellknown, post-gastrectomy syndrome that negatively affects patient quality of life (QOL) by causing hypoglycemia secondary to excess insulin secretion, following meal-induced hyperglycemia [2]. However, hypoglycemic symptoms, including general fatigue, cold sweat, and tremor, arise independently of the surgical procedure or the nature of the reconstruction performed. In addition, if patients do not report hypoglycemic symptoms, their blood glucose concentrations are routinely measured at most three times a day immediately before every meal prior to their discharge from hospital, and may not be measured at all after discharge. Therefore, postoperative blood glucose fluctuations are usually not fully documented. 
Continuous glucose monitoring (CGM) can be used to measure interstitial glucose concentration, which closely approximates plasma glucose concentration, after a short time-lag. CGM provides detailed information about the 24-h glycemic profile, including nocturnal and postprandial glycemia, which standard finger-prick blood glucose monitoring cannot provide [3]. Therefore, the use of CGM has the potential to provide a more comprehensive insight into the glycemic profile of patients following gastrectomy.

In the present study, we monitored 24-h interstitial glucose concentrations after gastrectomy to obtain information regarding glucose fluctuations, including these due to dumping syndrome, and to compare the glycemic profiles obtained in patients who had undergone distal gastrectomy (DG) or total gastrectomy (TG). At the same time, the patients' dumping symptoms and QOL were also evaluated using the Post-gastrectomy Syndrome Assessment Scale 37-item questionnaire (PGSAS-37).

\section{Materials and methods}

\section{Patients}

Between September 2017 and September 2019, a total of 107 patients with gastric cancer underwent curative TG or DG in our hospital. Of these, 70 patients were enrolled in this study, whereas the remaining 37 patients were excluded because of diabetes, because they also underwent resection of other organs, excluding cholecystectomy, or because of other organ malignancies or residual gastric cancer. Diabetes was defined as a casual blood glucose $\geq 200 \mathrm{mg} / \mathrm{dL}$ or HbAlc $\geq 6.5 \%$ [4]. In the TG group, simple Roux-en-Y (R-Y) reconstruction was performed, while in the DG group, Billroth-I (B-I) or R-Y reconstruction was performed. The type of reconstruction performed during DG was determined according to the experience of the surgeon. Body mass index, blood glucose, and $\mathrm{HbA1c}$ were evaluated before surgery. The clinical and pathological stages of the malignancies were determined on the basis of the 14th edition of the Japanese Classification of Gastric Carcinoma [5]. As a control group for the glucose monitoring, seven patients without diabetes who had undergone partial resection of the stomach because of submucosal tumors, during the same period, were also evaluated. The gastrectomy performed in these patients would have had a minimal effect on reservoir capacity and did not necessitate reconstruction.

\section{Glucose monitoring}

We used a CGM device to document post-gastrectomy glycemic profile. The FreeStyle Libre ${ }^{\circledR}$ Flash Glucose Monitoring System (Abbott Diabetes Care Inc., Alameda,
CA, USA) is a CGM device that provides a record of an individual's interstitial glucose concentrations, trends, and patterns. The sensor continuously measures glucose concentration in interstitial fluid through a small filament inserted just under the skin, records glucose concentrations every $15 \mathrm{~min}$ for up to 14 days, and displays the data when scanned. A Flash CGM sensor was placed subcutaneously on each patient's left upper arm immediately prior to discharge from hospital. The data were downloaded and analyzed using standard measures of amplitude and timing, including the mean, median, standard deviation (SD), maximum, minimum, and percentage of time within the target range (glucose concentration $<80 \mathrm{mg} / \mathrm{dL}$, $80-140 \mathrm{mg} / \mathrm{dL}$, or $>140 \mathrm{mg} / \mathrm{dL}$ ).

\section{Evaluation of post-gastrectomy syndromes}

To evaluate post-gastrectomy syndromes, including dumping syndrome, and QOL, we used the Post-gastrectomy Syndrome Assessment Scale 37-item questionnaire (PGSAS-37) developed by the Japan Post-gastrectomy Syndrome Working Party [6]. The main outcomes on the PGSAS-37 were recorded on seven symptom scales (esophageal reflux, abdominal pain, meal-related distress, indigestion, diarrhea, constipation, and dumping), four independent living status scales (amount of food ingested at each meal, necessity for additional food, quality of ingestion, ability to work), and one QOL scale (dissatisfaction in daily life). The total symptom score was calculated as the mean value for the seven symptom scales. In the PGSAS-37 questionnaire, high scores for the amount of food ingested per meal and the quality of ingestion reflected positive outcomes, whereas low scores for necessity for additional food, ability to work, and dissatisfaction in daily life reflected positive outcomes. The questionnaire was distributed to all the participants, who were instructed to complete it for the period between discharge and 1 month after the procedure, and then to return it to the department.

\section{Statistical analysis}

All statistical analyses were performed using JMP software (v. 13; SAS Institute, Cary, NC, USA). Continuous variables are presented as means \pm standard deviations and were analyzed using Student's $t$ or the Mann-Whitney $U$ test, while categorical variables are expressed as frequencies and were analyzed using Pearson's $\chi^{2}$ test. Spearman's correlation was used to assess the relationship between SD and the dumping symptom score. The statistical tests were two-sided and $P<0.05$ was considered to represent statistical significance. 


\section{Results}

\section{Patient demographics and characteristics}

A total of 57 patients had undergone DG and 13 had undergone TG (Table 1). The two groups were similar in terms of age, sex, body mass index, preoperative casual blood glucose concentration, and HbA1c. The TG group included more advanced cases, which necessitated open gastrectomy and longer postoperative hospital stays than the DG group.

\section{Glycemic profile after gastrectomy}

The glycemic profiles of representative patients are shown in Fig. 1. In the control group without diabetes, meal-induced increases in glucose concentration occurred, but hyperglycemia (glucose concentration $>140 \mathrm{mg} / \mathrm{dL}$ ) and hypoglycemia (glucose $<80 \mathrm{mg} / \mathrm{dL}$ ) were rare (Fig. 1a). Conversely, hypoglycemia was detected in patients with TG and DG after a postprandial glucose peak, which suggests late dumping syndrome (Fig. 1b, c). Glucose fluctuations were particularly evident in TG patients and nocturnal periods of hypoglycemia following the evening meal were also identified in both
Table 1 Patient demographics and characteristics
Fig. 1 Representative patient glycemic profiles. Profiles of: a a patient who had undergone partial resection of the stomach (control); b a patient who had undergone a distal gastrectomy (DG); and $\mathbf{c}$ a patient who had undergone a total gastrectomy (TG)

\begin{tabular}{llll}
\hline & DG $(n=57)$ & TG $(n=13)$ & $P$ value \\
\hline Age, years & $65.9 \pm 11.0$ & $66.7 \pm 10.7$ & 0.5967 \\
Gender, male/female & $29 / 28$ & $9 / 4$ & 0.2244 \\
Body mass index $\left(\mathrm{kg} / \mathrm{m}^{2}\right)$ & $21.6 \pm 3.1$ & $22.1 \pm 4.0$ & 0.6505 \\
Preoperative blood glucose level (mg/dL) & $101.9 \pm 11.2$ & $101.3 \pm 15.6$ & 0.8598 \\
Preoperative HbAlc (\%) & $5.6 \pm 0.4$ & $5.6 \pm 0.3$ & 0.7458 \\
Pathological T factor, T1/2/3/4 & $36 / 5 / 10 / 6$ & $5 / 1 / 2 / 5$ & 0.0081 \\
Pathological N factor, N0/1/2/3 & $37 / 12 / 3 / 5$ & $5 / 5 / 0 / 3$ & 0.2501 \\
Pathological stage, I/II/III/IV & $36 / 12 / 8 / 1$ & $6 / 1 / 5 / 1$ & 0.0090 \\
Approach, open/laparoscopic & $18 / 39$ & $11 / 2$ & 0.0010 \\
Postoperative hospital stay (days) & $12.9 \pm 8.6$ & $17.3 \pm 8.4$ & 0.0008 \\
\hline
\end{tabular}

Continuous variables are presented as means with standard deviation

$D G$ distal gastrectomy, $T G$ total gastrectomy

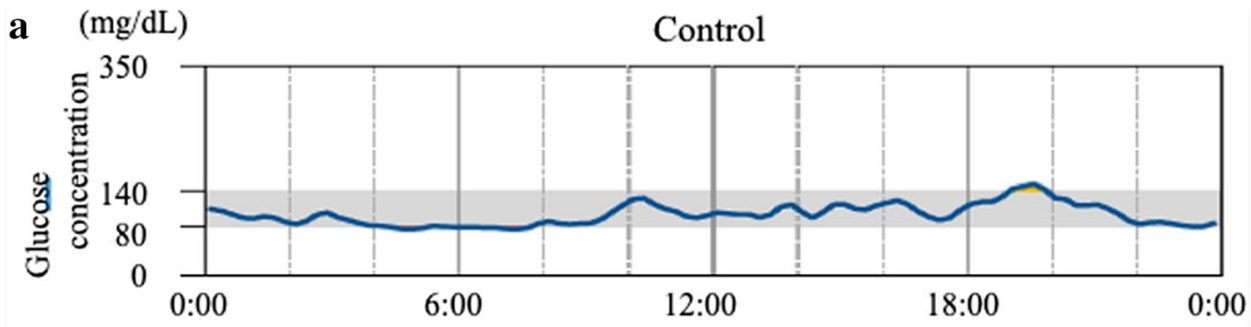

b $(\mathrm{mg} / \mathrm{dL}) \quad$ Patient with a distal gastrectomy

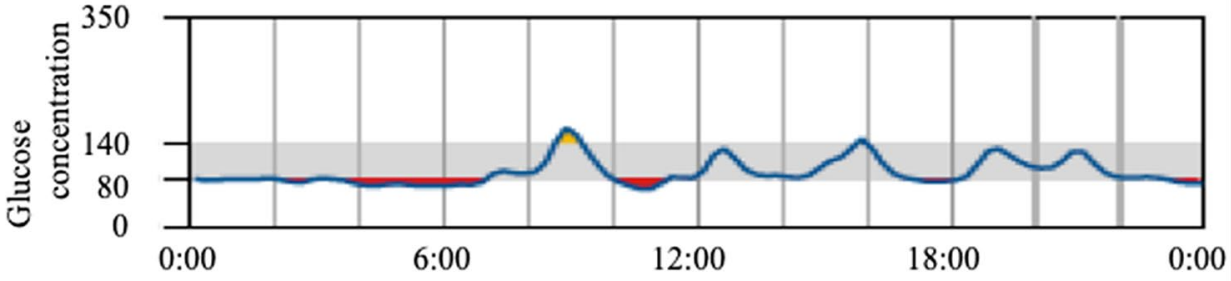

c $(\mathrm{mg} / \mathrm{dL}) \quad$ Patient with a total gastrectomy

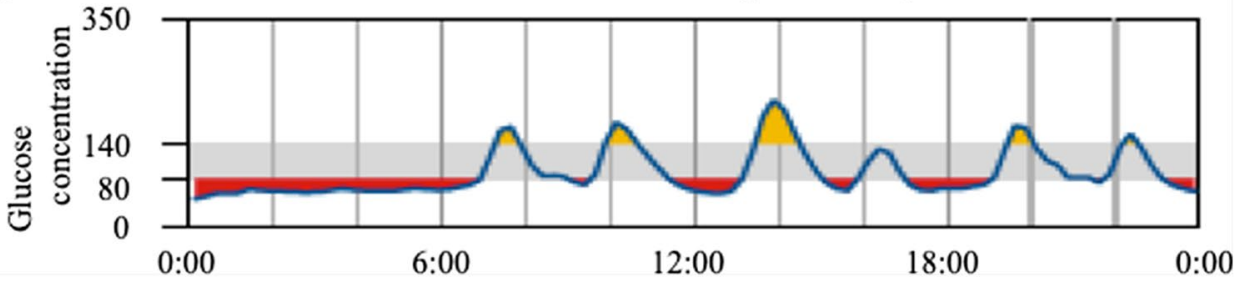


TG and DG patients. Nine of the 13 TG patients (69.2\%) and 32 of the 57 DG patients $(56.1 \%)$ showed nocturnal hypoglycemia of $<70 \mathrm{mg} / \mathrm{dL}$ for more than $20 \%$ of the time between 00:00 and 06:00 $\mathrm{h}$ (data not shown).

Glycemic variability can be evaluated using the SD of the CGM measurements. The two treatment groups had similar mean glucose concentrations (TG $85.0 \mathrm{vs}$. DG $89.1 \mathrm{mg} / \mathrm{dL}, P=0.2212$ ), which were lower than that of the control group $(99.5 \mathrm{mg} / \mathrm{dL})$. The DG and the control group had similar SDs, but the TG group d had a larger SD (TG 27.2 vs. DG $22.7 \mathrm{mg} / \mathrm{dL}, P=0.0551$ ) (Table 2). There were no significant differences between the maximum and minimum glucose values. In the TG group, $33.8 \%$ and $6.4 \%$ of the time the glucose values were $<70$ (generally used as the definition of hypoglycemia) and $>140 \mathrm{mg} / \mathrm{dL}$, respectively, while in the DG group, the respective percentages were $20.8 \%$ and $4.5 \%$ (TG vs. DG; $P=0.0454$ and 0.4800 , respectively) (Table 2). Both treatment groups, but especially the TG group, showed more frequent nocturnal hypoglycemia $(<70 \mathrm{mg} / \mathrm{dL}$ between 00:00 and 06:00 h;
TG 55.5 vs. DG $37.0 \%, P=0.0936)$, than the control group. The TG group had a lower mean glucose concentration (TG 67.5 vs. DG $74.9 \mathrm{mg} / \mathrm{dL}, P=0.0285$ ) than the DG group, but the mean glucose concentration of the DG group was also lower than that of the control group.

We also evaluated the effect of the type of reconstruction used in the DG group. R-Y reconstruction was associated with a lower mean glucose concentration (R-Y 85.2 vs. B-I $92.1 \mathrm{mg} / \mathrm{dL}, P=0.0282)$ and a higher percentage of time with glucose $<70 \mathrm{mg} / \mathrm{dL}$ (R-Y 27.7 vs. B-I $15.5 \%$, $P=0.0395$ ) than B-I reconstruction (Table 3). Nocturnal hypoglycemia was also more marked following R-Y reconstruction than B-I reconstruction (mean glucose concentration, R-Y $70.4 v s$. B-I $78.4 \mathrm{mg} / \mathrm{dL}, P=0.0254$; percentage of the time $<70 \mathrm{mg} / \mathrm{dL}, \mathrm{R}-\mathrm{Y} 48.8$ vs. $27.8 \%$, $P=0.0238$ ). There were no differences in either the DG or TG groups with respect to glycemic profile between patients who underwent open or laparoscopic approaches (data not shown).
Table 2 Standard measures and the percentages of time the participants had glucose concentrations of $>140 \mathrm{mg} / \mathrm{dL}$ or $<70 \mathrm{mg} / \mathrm{dL}$
Table 3 Standard measures and percentages of time the participants had glucose concentrations of $>140 \mathrm{mg} / \mathrm{dL}$ or $<70 \mathrm{mg} / \mathrm{dL}$ after DG

\begin{tabular}{|c|c|c|c|c|}
\hline & Control $(n=7)$ & DG $(n=57)$ & TG $(n=13)$ & $P$ value \\
\hline Mean glucose level (mg/dL) & $99.5 \pm 6.9$ & $89.1 \pm 11.7$ & $85.0 \pm 10.9$ & 0.2212 \\
\hline Standard deviation of glucose level (mg/dL) & $21.2 \pm 4.2$ & $22.7 \pm 4.8$ & $27.2 \pm 7.6$ & 0.0551 \\
\hline Maximum of glucose level (mg/dL) & $183.0 \pm 23.7$ & $189.6 \pm 31.8$ & $199.8 \pm 40.7$ & 0.5015 \\
\hline Minimum of glucose level (mg/dL) & $55.9 \pm 11.8$ & $45.3 \pm 7.9$ & $41.9 \pm 3.1$ & 0.3447 \\
\hline$\%$ time of $>140 \mathrm{mg} / \mathrm{dL}$ & $5.1 \pm 3.1$ & $4.5 \pm 3.6$ & $6.4 \pm 5.8$ & 0.4800 \\
\hline$\%$ time of $<70 \mathrm{mg} / \mathrm{dL}$ & $5.6 \pm 5.0$ & $20.8 \pm 20.3$ & $33.8 \pm 21.2$ & 0.0454 \\
\hline \multicolumn{5}{|l|}{ Nocturnal hypoglycemia } \\
\hline Mean glucose level (mg/dL) & $85.0 \pm 10.5$ & $74.9 \pm 12.8$ & $67.5 \pm 9.1$ & 0.0285 \\
\hline$\%$ time of $<70 \mathrm{mg} / \mathrm{dL}$ & $12.4 \pm 13.1$ & $37.0 \pm 32.4$ & $55.5 \pm 35.7$ & 0.0936 \\
\hline
\end{tabular}

Continuous variables are presented as means with standard deviations. "Nocturnal hypoglycemia" refers to data collected between 00:00 and 06:00 h. The $P$ values refer to comparisons of DG and TG

$D G$ distal gastrectomy, $T G$ total gastrectomy

\begin{tabular}{lcccc}
\hline & Control $(n=7)$ & B-I $(n=32)$ & R-Y $(n=25)$ & $P$ value \\
\hline Mean glucose level (mg/dL) & $99.5 \pm 6.9$ & $92.1 \pm 10.9$ & $85.2 \pm 11.6$ & 0.0282 \\
Standard deviation of glucose (mg/dL) & $21.2 \pm 4.2$ & $22.5 \pm 4.4$ & $23.0 \pm 5.3$ & 0.6936 \\
Maximum of glucose level (mg/dL) & $183.0 \pm 23.7$ & $191.9 \pm 31.5$ & $186.7 \pm 32.7$ & 0.5304 \\
Minimum of glucose level (mg/dL) & $55.9 \pm 11.8$ & $47.2 \pm 8.5$ & $42.8 \pm 6.4$ & 0.0107 \\
\% time of $>140 \mathrm{mg} / \mathrm{dL}$ & $5.1 \pm 3.1$ & $5.3 \pm 4.1$ & $3.6 \pm 2.6$ & 0.1588 \\
$\%$ time of $<70 \mathrm{mg} / \mathrm{dL}$ & $5.6 \pm 5.0$ & $15.5 \pm 17.0$ & $27.7 \pm 22.4$ & 0.0395 \\
Nocturnal hypoglycemia & & & & \\
Mean glucose level (mg/dL) & $85.0 \pm 10.5$ & $78.4 \pm 10.3$ & $70.4 \pm 14.4$ & 0.0254 \\
$\%$ time of $<70 \mathrm{mg} / \mathrm{dL}$ & $12.4 \pm 13.1$ & $27.8 \pm 27.1$ & $48.8 \pm 35.3$ & 0.0238 \\
\hline
\end{tabular}

Continuous variables are presented as means with standard deviations. "Nocturnal hypoglycemia" refers to data collected between 00:00 and 06:00 h. The $P$ values refer to comparisons of B-I and R-Y reconstructions

$B-I$ Billroth-I reconstruction, $R-Y$ Roux-en-Y reconstruction, $D G$ distal gastrectomy 


\section{Dumping syndrome, postoperative symptoms, and quality of life}

The PGSAS-37 symptom scores 1 month after surgery are listed in Table 4 . The dumping score was significantly higher, reflecting a poorer outcome, in the TG group than the DG group (TG 2.4 vs. DG 1.3, $P=0.0061$ ). Meal-related distress, diarrhea, and the ability to work scores were also higher in the TG group. However, the QOL (dissatisfaction in daily life) score was similar in the two groups.

Next, we analyzed the relationship between the dumping symptom score and the SD of the glucose concentrations, reflecting glycemic variability, and found no correlation between dumping symptom score and glycemic variability ( $\rho=0.0545, P=0.6662$ ). (Fig. 2). This implies that dumping symptoms do not necessarily reflect glucose fluctuations or hypoglycemia.

\section{Discussion}

Glucose fluctuation after gastrectomy, represented by dumping syndrome, is a post-gastrectomy syndrome that impairs patient QOL, the mechanism of which has been previously elucidated [2]. To avoid hypoglycemia following postprandial hyperglycemia, patients who undergo gastrectomy for gastric cancer routinely receive advice to eat slowly; to eat smaller and more frequent meals; to avoid ingesting fluids with their meals; to limit sugary food intake; to eat more protein, complex carbohydrates, and fiber; and to eat high-sugar foods immediately if they identify signs of hypoglycemia. Patients undergoing gastrectomy are rarely administered insulin for hyperglycemia or glucose for hypoglycemia in our hospital, because there has been an assumption that the

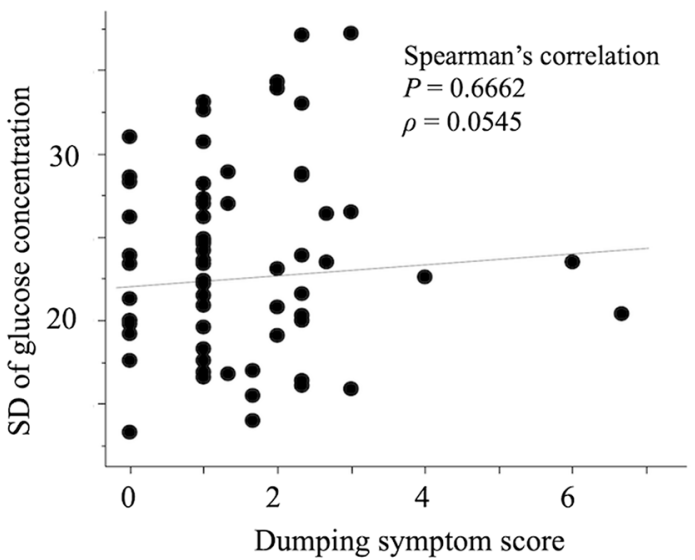

Fig. 2 Relationship between the standard deviation (SD) of the glucose concentration and the dumping symptom score. SD is indicative of glycemic variability. There was no correlation between the dumping symptom score and glycemic variability

dietary recommendations are effective and that patients do not develop dumping syndrome. In the present study, we continuously measured subcutaneous glucose concentration in patients that had undergone gastrectomy using a CGM system, and analyzed the values with reference to an objective evaluation of dumping, made using the PGSAS-37 questionnaire. The results showed that the diurnal glycemic variability is greater, nocturnal hypoglycemia is more frequent than expected, and some patients develop hypoglycemia without showing hypoglycemic symptoms. To our knowledge, this is the first study to systematically characterize the post-gastrectomy glycemic profile using a CGM system.

Glycemic variability and post-gastrectomy symptoms were more severe in patients who had undergone TG. The largest difference between TG and DG is the difference in
Table 4 Post-gastrectomy Syndrome Assessment Scale 37-item questionnaire scores 1 month after gastrectomy

\begin{tabular}{lllll}
\hline Domains & Main outcome measures & DG $(n=57)$ & TG $(n=13)$ & $P$ value \\
\hline \multirow{2}{*}{ Symptoms } & Esophageal reflux & $2.2 \pm 1.2$ & $2.1 \pm 0.9$ & 0.9118 \\
& Abdominal pain & $2.3 \pm 1.1$ & $2.2 \pm 0.9$ & 0.9120 \\
& Meal-related distress & $2.6 \pm 1.4$ & $3.6 \pm 1.1$ & 0.0094 \\
& Indigestion & $2.0 \pm 1.0$ & $2.2 \pm 0.8$ & 0.3153 \\
& Diarrhea & $1.7 \pm 1.1$ & $2.9 \pm 1.5$ & 0.0053 \\
& Constipation & $2.6 \pm 1.3$ & $2.6 \pm 1.8$ & 0.6491 \\
\multirow{4}{*}{ Living status } & Dumping & $1.3 \pm 1.2$ & $2.4 \pm 1.4$ & 0.0061 \\
& Total symptom score & $2.1 \pm 0.7$ & $2.6 \pm 0.8$ & 0.0299 \\
& Ingested amount of food per meal & $3.4 \pm 2.1$ & $3.8 \pm 2.2$ & 0.6178 \\
& Necessity for additional food & $2.4 \pm 1.1$ & $3.3 \pm 1.5$ & 0.0485 \\
& Quality of ingestion & $2.5 \pm 1.2$ & $3.2 \pm 0.9$ & 0.0773 \\
QOL & Ability for working & $2.7 \pm 1.4$ & $3.9 \pm 1.3$ & 0.0120 \\
\hline
\end{tabular}

Data are presented as means with standard deviations

$D G$ distal gastrectomy, $T G$ total gastrectomy, $Q O L$ quality of life 
the reservoir capacity of the remnant stomach. Therefore, surgeons have used several methods to add capacity during the reconstruction following TG. For example, reconstruction with a jejunal pouch compensates for the loss of reservoir capacity and reportedly reduces the severity of symptoms related to the dumping syndrome or reflux, resulting in an improvement in QOL [7-9]. In addition, aboral pouch reconstruction, in which a jejunal pouch is created in the $Y$ limb of a Roux-en-Y (R-Y) reconstruction, has been reported to be associated with significantly higher serum cholesterol concentrations and QOL than simple R-Y reconstruction [10]. However, jejunal pouch reconstruction is complex and can sometimes be associated with food stasis and excessive pouch dilatation [11], such that Tanaka et al. [12] reported no benefit of the aboral pouch procedure in their randomized trial. Therefore, we currently perform a simple R-Y reconstruction after TG, which remains the standard technique [13], and the larger glycemic variability and more severe symptoms in the TG group are consistent with other previous reports [14-16].

The PGSAS-37 questionnaire data show that patients with TG often experience meal-related distress and diarrhea, in addition to dumping syndrome. These symptoms could also promote hypoglycemia. However, the most important problems identified were the nocturnal hypoglycemia following both TG and DG and the diurnal glycemic variability in many patients who had undergone TG, which were frequently not reflected in symptoms. Indeed, the patients whose data are shown in Fig. 1b, c did not report hypoglycemic symptoms. There was no correlation between dumping symptom score and glycemic variability (evaluated using the SD) ( $\rho=0.0545, P=0.6662)$ (Fig. 2). Furthermore, it is likely that patients did not detect their hypoglycemia while asleep. Thus, there is a possibility that glucose variability and hypoglycemia can occur for reasons other than the dumping syndrome in patients who do not have diabetes. Asymptomatic hypoglycemia, including nocturnal hypoglycemia unawareness, is likely to manifest as repeated episodes of mild hypoglycemia. This results in a weakening of the autonomic response, termed 'hypoglycemia-associated autonomic failure', which further contributes to the development of hypoglycemia unawareness [17, 18].

In our study, there was a difference in glycemic profile in patients that had undergone B-I or R-Y reconstructions after DG. Overall, R-Y reconstruction was associated with a lower mean glucose concentration and more frequent hypoglycemia of $(<70 \mathrm{mg} / \mathrm{dL})$, but the glycemic variability was similar following each of the two reconstructions. However, the choice of reconstruction was not randomized, instead being determined according to the surgeon's experience, because there is no specific indication for B-I or R-Y reconstruction. However, the remnant stomach was probably smaller following R-Y reconstruction, because this tended to be performed when the tumor was located on upper side of the distal stomach. The size of the remnant stomach could have affected the glycemic profile after gastrectomy. Following TG, the complete loss of reservoir capacity may be a cause of the larger glycemic variability.

In addition, the reconstruction method may affect the risk of hypoglycemia. Choi et al. [19] compared the effects of R-Y and B-I reconstruction after DG on diabetes control in patients with early gastric cancer who had type 2 diabetes, and showed that R-Y reconstruction specifically ameliorated type 2 diabetes. Costa et al. [20] also found that $\mathrm{R}-\mathrm{Y}$ reconstruction significantly reduced BMI and ameliorated diabetes in obese patients with type 2 diabetes. Thus, $\mathrm{R}-\mathrm{Y}$ reconstruction seems to be more effective at lowering blood glucose than other methods of reconstruction. In addition, Takase et al. [21] suggested that a reconstruction that permitted the passage of food through the duodenum was associated with better digestion and absorption, resulting in superior physical status. R-Y reconstruction has also been favored for the prevention of bile reflux and the development of remnant gastric cancer [22, 23]. Thus, each reconstruction method after DG has advantages and disadvantages, and it appears that the reconstruction method has an influence on glucose metabolism. We are now conducting further investigations into the effect of the type of reconstruction after gastrectomy on subsequent glucose concentrations.

In recent years, glucose fluctuations and nocturnal hypoglycemia have become recognized as specific causes of complications in diabetic patients, but the importance of these phenomena post-gastrectomy has not been determined to date. Hypoglycemia not only leads to a shortterm decline in QOL but also has long-term negative effects, such as inducing greater risks of cardiovascular events and mortality [24, 25]. A relationship between hypoglycemia and dementia, particularly in older patients, has also been reported recently [26, 27], and this is likely to become increasingly significant, given the aging population. Furthermore, it has become apparent that not only hyperglycemia and high $\mathrm{HbA1c}$ concentrations, but also glycemic variability, can be associated with negative outcomes. Glycemic variability has been reported to be a strong independent risk factor for mortality in critically ill patients [28, 29], and Nusca et al. [30] have suggested that glycemic variability is a potential risk factor for the development of cardiac complications in both diabetic and non-diabetic patients. Furthermore, Akirov et al. [31] have reported that in patients with or without diabetes mellitus who are hospitalized in general surgery wards, high glycemic variability is associated with longer hospitalization and higher short-term and long-term mortality. These findings imply that surgeons should be concerned about the degree of glycemic variability after gastrectomy, in addition to hypoglycemia, not only with regard to the 
short-term outcomes and QOL, but also with regard to the long-term outcomes. Rather than DG, a pylorus-preserving gastrectomy; and rather than TG, a proximal gastrectomy or subtotal gastrectomy with the preservation of a small stomach remnant if possible; may be preferable for the avoidance of glucose variability and hypoglycemia.

Some potential limitations of the present study should be acknowledged. First, we measured glucose concentration and patients completed the PGSAS-37 questionnaire over only the first month following gastrectomy, during which patient food intake and symptoms are usually variable. In addition, patients with Stage II or higher tumors require adjuvant chemotherapy within the first 6 weeks after gastrectomy, which could greatly influence their food intake and QOL [32], meaning that the study had to be performed before the participants underwent chemotherapy. However, the most important point is that surgeons should be aware of the fact that substantial glycemic variability and high frequent hypoglycemia occur at that time. Second, this was a single-institute study and the sample size was small, especially in the TG group. Third, patient food intake was not standardized during the glucose monitoring, because the Flash CGM sensor was placed immediately prior to discharge from hospital. All the patients received dietary advice from a dietician before discharge, and we subsequently collected questionnaires in which patients completed details of their meal composition and timing. However, it was difficult to accurately evaluate energy, protein, fat, and carbohydrate intake. Therefore, we cannot rule out the differences between the groups being due to differences in food intake, and the present findings should be validated in a larger group of patients, using standardized approaches.

In conclusion, the use of a CGM system is valuable for the assessment of glycemic profile after gastrectomy. It permits the recognition of diurnal glycemic variability and nocturnal hypoglycemia in patients undergoing gastrectomy. In addition, it has also revealed that the glycemic profile differs, depending on the reconstruction method used after DG. Finally, because some hypoglycemic patients did not report symptoms and glycemic variability did not necessarily correlate with the presence of dumping symptoms, dumping syndrome appears to explain only a fraction of the postoperative glucose fluctuations present during the course of a day.

Acknowledgements This work was supported in part by a Grant-in Aid for Scientific Research, No 18H02882. We thank Mark Cleasby from Edanz Group (www.edanzediting.com/ac) for editing drafts of this manuscript.

\section{Compliance with ethical standards}

Conflict of interest The authors declare that they have no conflicts of interest.
Ethical standards This study conformed to the ethical guidelines of the World Medical Association Declaration of Helsinki. All patients gave their written informed consent for surgery and the use of clinical data, as required by the Institutional Review Board of the Kyoto Prefectural University of Medicine (ERB-C-975-2).

\section{References}

1. Bray F, Ferlay J, Soerjomataram I, Siegel RL, Torre LA, Jemal A. Global cancer statistics 2018: GLOBOCAN estimates of incidence and mortality worldwide for 36 cancers in 185 countries. CA Cancer J Clin. 2018;68:394-424.

2. Holdsworth CD, Turner D, McIntyre N. Pathophysiology of postgastrectomy hypoglycaemia. Br Med J. 1969;4:257-9.

3. Bolinder J, Antuna R, Geelhoed-Duijvestijn P, Kröger J, Weitgasser R. Novel glucose-sensing technology and hypoglycaemia in type 1 diabetes: a multicentre, non-masked, randomised controlled trial. Lancet. 2016;388:2254-63.

4. Seino Y, Nanjo K, Tajima N, Kadowaki T, Kashiwagi A, Araki E, Ito C, Inagaki N, Iwamoto Y, Kasuga M, Hanafusa T, Haneda M, Ueki K. Report of the committee on the classification and diagnostic criteria of diabetes mellitus. Committee of the Japan Diabetes Society on the Diagnostic Criteria of Diabetes Mellitus. J Diabetes Investig. 2010;1:212-28.

5. Japanese Gastric Cancer Association. Japanese classification of gastric carcinoma: 3rd English edition. Gastric Cancer. 2011;14:101-12.

6. Nakada K, Ikeda M, Takahashi M, Kinami S, Yoshida M, Uenosono Y, Kawashima Y, Oshio A, Suzukamo Y, Terashima M, Kodera Y. Characteristics and clinical relevance of postgastrectomy Syndrome Assessment Scale (PGSAS)-45: newly developed integrated questionnaires for assessment of living status and quality of life in postgastrectomy patients. Gastric Cancer. 2015;18:147-58.

7. Nakane Y, Okumura S, Akehira K, Okamura S, Boku T, Okusa $\mathrm{T}$, et al. Jejunal pouch reconstruction after total gastrectomy for cancer. A randomized controlled trial. Ann Surg. 1995;222:27-35.

8. Fein M, Fuchs KH, Thalheimer A, Freys SM, Heimbucher J, Thiede A. Long-term benefits of Roux-en-Y pouch reconstruction after total gastrectomy: a randomized trial. Ann Surg. 2008;247:759-65.

9. Gertler R, Rosenberg R, Feith M, Schuster T, Friess H. Pouch vs. no pouch following total gastrectomy: meta-analysis and systematic review. Am J Gastroenterol. 2009;104:2838-51.

10. Horvath OP, Kalmar K, Cseke L, Poto L, Zambo K. Nutritional and life-quality consequences of aboral pouch construction after total gastrectomy: a randomized, controlled study. Eur J Surg Oncol. 2001;27:558-63.

11. Tono C, Terashima M, Takagane A, Abe K. Ideal reconstruction after total gastrectomy by the interposition of a jejunal pouch considered by emptying time. World J Surg. 2003;27:1113-8.

12. Tanaka C, Kanda M, Murotani K, Yoshikawa T, Cho H, Ito Y, Matsui T, Nakayama H, Yamada T, Kobayashi D, Fujiwara M, Kodera Y. Long-term quality of life and nutrition status of the aboral pouch reconstruction after total gastrectomy for gastric cancer: a prospective multicenter observational study (CCOG1505). Gastric Cancer. 2019;22:607-16.

13. Syn NL, Wee I, Shabbir A, Kim G, So JB. Pouch versus no pouch following total gastrectomy: meta-analysis of randomized and non-randomized studies. Ann Surg. 2019;269:1041-53.

14. Kurihara K, Tamai A, Yoshida Y, Yakushiji Y, Ueno H, Fukumoto M, Hosoi M. Effectiveness of sitagliptin in a patient with late 
dumping syndrome after total gastrectomy. Diabetes Metab Syndr. 2018;12:203-6.

15. Fujita Y, Tamada D, Kozawa J, Kobayashi Y, Sasaki S, Kitamura T, Yasuda T, Maeda N, Otsuki M, Okita K, Iwahashi H, Kaneto H, Funahashi T, Imagawa A, Shimomura I. Successful treatment of reactive hypoglycemia secondary to late dumping syndrome using miglitol. Intern Med. 2012;51:2581-5.

16. Tanizawa Y, Tanabe K, Kawahira H, Fujita J, Takiguchi N, Takahashi M, Ito Y, Mitsumori N, Namikawa T, Oshio A, Nakada K, Japan Postgastrectomy Syndrome Working Party. Specific features of dumping syndrome after various types of gastrectomy as assessed by a newly developed integrated questionnaire, the PGSAS-45. Dig Surg. 2016;33:94-103.

17. Heller SR, Cryer PE. Reduced neuroendocrine and symptomatic responses to subsequent hypoglycemia after 1 episode of hypoglycemia in nondiabetic humans. Diabetes. 1991;40:223-6.

18. Cryer PE. Hypoglycemia-associated autonomic failure in diabetes. Am J Physiol Endocrinol Metab. 2001;281:E1115-E112121.

19. Choi YY, Noh SH, An JY. A randomized controlled trial of Rouxen-Y gastrojejunostomy vs. gastroduodenostomy with respect to the improvement of type 2 diabetes mellitus after distal gastrectomy in gastric cancer patients. PLoS ONE. 2017;12:e0188904.

20. Costa M, Trovão Lima A, Morais T, Almeida RF, Nora M, Guimarães M, Monteiro MP. Does reconstruction type after gastric resection matters for type 2 diabetes improvement? J Gastrointest Surg. 2019. https://doi.org/10.1007/s11605-019-04255-4.

21. Takase M, Sumiyama Y, Nagao J. Quantitative evaluation of reconstruction methods after gastrectomy using a new type of examination: digestion and absorption test with stable isotope 13C-labeled lipid compound. Gastric Cancer. 2003;6:134-41.

22. Shinoto K, Ochiai T, Suzuki T, Okazumi S, Ozaki M. Effectiveness of Roux-en-Y reconstruction after distal gastrectomy based on an assessment of biliary kinetics. Surg Today. 2003;33(3):169-77.

23. Kojima K, Yamada H, Inokuchi M, Kawano T, Sugihara $\mathrm{K}$. A comparison of Roux-en-Y and Billroth-I reconstruction after laparoscopy-assisted distal gastrectomy. Ann Surg. 2008;247(6):962-7.
24. ORIGIN Trial Investigators, Mellbin LG, Rydén L, Riddle MC, Probstfield J, Rosenstock J, Díaz R, Yusuf S, Gerstein HC. Does hypoglycaemia increase the risk of cardiovascular events? A report from the ORIGIN trial. Eur Heart J. 2013;34:3137-44.

25. International Hypoglycaemia Study Group. Hypoglycaemia, cardiovascular disease, and mortality in diabetes: epidemiology, pathogenesis, and management. Lancet Diabetes Endocrinol. 2019;7:385-96 (Review).

26. Whitmer RA, Karter AJ, Yaffe K, Quesenberry CP Jr, Selby JV. Hypoglycemic episodes and risk of dementia in older patients with type 2 diabetes mellitus. JAMA. 2009;301:1565-72.

27. Mehta HB, Mehta V, Goodwin JS. Association of hypoglycemia with subsequent dementia in older patients with type 2 diabetes mellitus. J Gerontol A Biol Sci Med Sci. 2017;72:1110-6.

28. Krinsley JS. Glycemic variability: a strong independent predictor of mortality in critically ill patients. Crit Care Med. 2008;36:3008-133.

29. Thomas F, Signal M, Chase JG. Using continuous glucose monitoring data and detrended fluctuation analysis to determine patient condition: a review. J Diabetes Sci Technol. 2015;9:1327-35.

30. Nusca A, Tuccinardi D, Albano M, Cavallaro C, Ricottini E, Manfrini S, et al. Glycemic variability in the development of cardiovascular complications in diabetes. Diabetes Metab Res Rev. 2018;34:e3047.

31. Akirov A, Shochat T, Dotan I, Diker-Cohen T, Gorshtein A, Shimon I. Glycemic variability and mortality in patients hospitalized in general surgery wards. Surgery. 2019;166:184-92.

32. Sakuramoto S, Sasako M, Yamaguchi T, Kinoshita T, Fujii M, Nashimoto A, Furukawa H, Nakajima T, Ohashi Y, Imamura H, Higashino M, Yamamura Y, Kurita A, Arai K, ACTS-GC Group. Adjuvant chemotherapy for gastric cancer with S-1, an oral fluoropyrimidine. N Engl J Med. 2007;357:1810-20.

Publisher's Note Springer Nature remains neutral with regard to jurisdictional claims in published maps and institutional affiliations. 